\title{
Etude des effets des éclaircies sur la qualité du bois de Cedrus atlantica Manetti
}

\author{
M. EL RHAZI (*) \\ I.N.R.A. Station de Recherches sur la Qualité des Bois \\ Centre de Recherches forestières de Nancy/Champenoux - F 54280 Seichamps
}

\begin{abstract}
Thèse de Docteur-Ingénieur - Université de Nancy I
15 mai 1981
\end{abstract}

Le but de l'étude est d'examiner les effets des modalités d'éclaircies d'intensités croissantes sur la structure du bois de cèdre de l'Atlas.

Ces traitements sylvicoles ne peuvent être appliqués que sur des stations où le cèdre vient bien et se régénère naturellement. Dans ces stations, on dispose généralement d'un nombre suffisant de tiges à l'hectare (2 500 à 3000 tiges) pour procéder à des éclaircies sans mettre en cause l'existence du peuplement.

Le dispositif d'éclaircies est situé dans la forêt du Seheb d'Azrou (Maroc) sur un sol brun très fertile, sur une roche-mère basaltique, avec une pluviosité abondante et une altitude moyenne pour le cèdre $(1750 \mathrm{~m})$.

Nous avons étudié quatre niveaux d'éclaircie $(* *)$ : un peuplement témoin (T) comportant 2600 tiges à l'hectare, puis trois peuplements où il subsiste après une éclaircie moyenne (M) 1460 tiges à l'hectare, une éclaircie forte (F) : 900 tiges à l'hectare, et enfin une éclaircie très forte (TF) : 320 tiges à l'hectare.

Des carottes de sondage ont été prélevées dans les arbres des différentes modalités. Cet échantillonnage non destructif a servi à déterminer les caractères de la qualité du bois : infradensité, composantes de la densité, largeur de cernes, les retraits dans les trois directions : axial, tangentiel, radial, et volumétrique. La méthode d'étude aux ultra-sons permet aussi de déterminer dans les trois directions les modules de Young $\left(\mathrm{E}_{\mathrm{L}}, \mathrm{E}_{\mathrm{T}}, \mathrm{E}_{\mathrm{l}}\right)$ qui sont liés aux caractéristiques mécaniques du bois. Nous

(*) Ingénieur des Eaux et Forêts au Centre de Technologic Forestière de Rabat (Maroc).

(**) Taux d'éclaircie par rapport au témoin : $(\mathrm{M})=44 \%,(\mathrm{~F})=66 \%,(\mathrm{TF})=88 \%$. 
n'avons considéré sur les carottes de sondage que les 15 derniers accroissements annuels correspondant au bois produit à la suite du dernier traitement d'éclaircie qui a amené les taux d'éclaircies aux valeurs données ci-dessus.

Les résultats obtenus sont :

- la largeur des cernes est différente entre les traitements et supérieure pour TF et $\mathrm{F}$, ce qui était attendu ;

- l'infradensité et les retraits ne sont pas différents entre les traitements, ce qui est un signe de conservation de qualité du bois ;

- les modules de Young $\mathrm{E}_{\mathrm{L}}, \mathrm{E}_{\mathrm{I} i}$ sont augmentés par les éclaircies $F$, $T F$, et l'éclaircie $F$ apporte l'effet le plus marqué. Le module $E_{R}$ n'est pas différent;

- l'examen détaillé des composantes de la densité montre que les moyennes de la densité minimum et de la densité maximum des accroissements annuels sont augmentées dans $F$ et TF, alors que les paliers de densité intermédiaire $(400-500)$, $(500-600),(600-700) \mathrm{kg} / \mathrm{m}^{3}$ ne sont pas différents. Ceci traduit une plus grande régularité des variations de densité du bois dans les accroissements annuels.

Les éclaircies successives n'ayant pas été appliquées régulièrement dans le temps, nous avons observé le phénomène de l'accélération de croissance : succession d'accroissements annuels larges et étroits, ainsi que l'alternance qui en est la répétition et qui sont néfastes à la qualité du bois.

Les traitements d'éclaircies appliqués au cèdre, et particulièrement le traitement $\mathrm{F}$ : $66 \%, 900$ tiges à l'hectare, contribuent à élever encore la qualité du bois de cèdre. Ils permettent d'obtenir des tiges de plus fort diamètre et de plus grande résistance mécanique. Si l'élagage artificiel de branches vivantes était appliqué d'une façon générale dans ces peuplements éclaircis, il devrait contribuer à obtenir plus de tiges sans nœuds destinées au déroulage ou au tranchage, comme cela se pratique actuellement sur une petite échelle. 